\title{
Réplique de Monsieur Marcel Bellavance à propos du compte rendu de son volume Un village en mutation : Compton, Québec, 1880-1920 par Jack I. Little, paru dans le numéro de juin $1983(37,1)$ de la $R H A F$, p. 92.
}

\section{Marcel Bellavance}

Volume 37, numéro 3, décembre 1983

URI : https://id.erudit.org/iderudit/304196ar

DOI : https://doi.org/10.7202/304196ar

Aller au sommaire du numéro

Éditeur(s)

Institut d'histoire de l'Amérique française

ISSN

0035-2357 (imprimé)

1492-1383 (numérique)

Découvrir la revue

Citer ce document

Bellavance, M. (1983). Réplique de Monsieur Marcel Bellavance à propos du compte rendu de son volume Un village en mutation : Compton, Québec,

1880-1920 par Jack I. Little, paru dans le numéro de juin $1983(37,1)$ de la $R H A F$, p. 92. Revue d'histoire de l'Amérique française, 37(3), 474-477.

https://doi.org/10.7202/304196ar 
Réplique de Monsieur Marcel Bellavance à propos du compte rendu de son volume Un village en mutation: Compton, Québec, 1880-1920 par Jack I. Little, paru dans le numéro de juin $1983(37,1)$ de la $R H A F$, p. 92.

Dans le numéro de juin 1983 de la Revue d'Histoire de l'Amérique française paraissait un compte rendu de ma monographie intitulée: Un village en mutation: Compton, Québec, 1880-1920, Parcs Canada, 1982. Ce texte de M. J.I. Little dont l'ordonnance des idées est souvent floue pêche au double niveau de l'objectivité et de la connaissance du sujet. Ces deux qualités nécessaires à la réalisation d'un tel exercice auraient permis dans un cas de rendre fidèlement compte de ce qui était écrit et, dans l'autre, de critiquer le sujet et la méthodologie utilisée. Le texte de M. Little ne possède malheureusement pour moi ni l'une ni l'autre. Je m'explique.

Mais avant, de quoi parlons-nous exactement? Un village en mutation ... est une monographie sur une petite communauté des Cantons de l'Est qui connut au début de ce siècle un renversement complet de sa structure démographique et passa ainsi en moins de dix ans de majorité anglaise à majorité française. L'ouvrage se divise en deux parties: l'une consacrée à la colonisation et aux transformations de l'économie dans les Cantons de l'Est au XIX ${ }^{\mathrm{e}}$ siècle, l'autre, plus spécifique, au village lui-même. Cette partie comprend six chapitres où sont abordés successivement des thèmes comme la population (effectifs démographiques, mouvements annuels et saisonniers...), l'environnement villageois, la propriété, la structure sociale et économique et, enfin, la vie culturelle (pratique religieuse, comportements politiques...). Ceci étant dit, venons-en au compte rendu de Little qui, d'entrée de jeu, écrit que j'ai «passé sous silence la colonisation canadienne-française du Canton de Compton» où est situé, on le devinera le village de Compton. Cela commence mal. Car on comprendra aisément qu'il ne pouvait en être autrement puisque les Canadiens français ne colonisèrent jamais cette région où étaient établis les Anglophones depuis le début du XIX ${ }^{\mathrm{e}}$ siècle. Les Canadiens français vinrent beaucoup plus tard dans cette région pour y acheter les terres et les propriétés des premiers occupants. Si Little insinue par là qu'il y eut absence d'analyse 
démographique dans le canton, je lui suggère une relecture de la monographie. Il verrait alors que, dans l'analyse des données statistiques comme les registres d'état civil, les recensements, les rôles d'évaluation, l'enregistrement des titres de propriété, les rapports pastoraux qu'il juge en passant «périmés», le rapport village-canton est bien fait. Un coup d'oeil sur les graphiques et les tableaux en font foi.

J'aurais, semble-t-il aussi, «déprécié le rôle stimulateur de l'Église et du Gouvernement» dans la colonisation des Cantons de l'Est. Nuancer une interprétation est-il la déprécier? C'est ainsi qu'il aurait fallu comprendre l'analyse de ce rôle que résume bien le paragraphe suivant pris à la page 10 de mon texte: «De telles mesures (abolition des réserves du clergé protestant, loi de protection du colon etc.) facilitèrent la pénétration canadienne-française dans les bonnes régions agricoles. Elles ne peuvent expliquer à elles seules, cependant, la rapidité avec laquelle elle s'est faite dans les Cantons de l'Est après 1850. L'action des Sociétés de colonisation et la législation ne viendraient qu'après coup aider un mouvement migratoire rendu irréversible par les pressions démographiques et une conjoncture économique favorable.»Comprenne qui veut.

Les Canadiens français, selon Little toujours, se seraient établis «sur le territoire en bordure des prospères villages anglophones». Little devrait savoir que les Anglophones ne colonisèrent jamais vraiment que la partie sud-ouest des Cantons comme l'indiquent les figures 1 et 3 (p. 8, 15) qu'il aurait dû encore une fois examiner attentivement. Les Francophones, contrairement, finirent par essaimer partout en colonisant les terres vierges et en s'appropriant ailleurs le domaine bâti. La thèse de Jean I. Hunter (The French Invasion of the Eastern Townships...) a clairement décrit ce phénomène et a démontré justement la tendance des Canadiens français à prendre d'abord contrôle des villages et municipalités en régions habitées avant d'occuper majoritairement la campagne. Le cas de Compton confirme cette interprétation.

Poursuivons l'examen de ce compte rendu. Que lisons-nous? «Sans doute que, vers la fin du XIX ${ }^{\mathrm{e}}$ siècle, les anciens propriétaires de seigneuries se seraient portés acquéreurs de certaines propriétés des Anglo-protestants, même si cette région fut peu habitée: ce sont surtout les Beaucerons qui émigrèrent (sic) en pays comptonien.» Que signifie cela? Que viennent bien faire les seigneurs dans cette galère? Quel est leur rapport aux Beaucerons de la fin du $\mathrm{XIX}^{\mathrm{e}}$ siècle qui migrèrent en pays comptonien?

Tout de suite après, Little écrit: «Néanmoins, les colonisateurs canadiensfrançais avaient réussi à implanter dans la région leur religion et leurs coutumes, ce qui contribua à rendre étonnamment souple la future transition ethnique des vieux cantons.» M. Little a vraiment l'art du coq à l'âne et essaie de donner encore ici une apparence logique aux idées qu'il est seul à comprendre. En effet, cette relation causale entre l'implantation d'une culture et la souplesse «étonnante» de la transition ethnique me paraît douteuse et relève de la pure ratiocination. Toujours au sujet du changement démographique, Little me demande "pourquoi l'émigration fut si rapide à Compton, alors que les communautés avoisinantes conservèrent beaucoup plus longtemps leur majorité anglophone»? Tentant lui-même une réponse, Little fait référence à l'«occupation des terres de l'Alberta» où s'établirent effectivement d'anciens 
résidants de Compton. Malheureusement, cela est insuffisant. J'ajouterai, quant à moi, que par rapport à l'ensemble des Cantons de l'Est, le mouvement migratoire comptonien s'est fait très tardivement puisque les Cantons de l'Est sont majoritairement français depuis déjà 1871 (p. 14). Quant à savoir pourquoi les villages et les cantons du sud-ouest dont Compton restèrent plus longtemps anglophones, il faut peut-être y voir la proximité de la frontière américaine et la concentration historique du groupe dans cette région où il subsiste toujours. Seules d'autres monographies régionales apporteraient quelque éclairage nouveau là-dessus.

Que lisons-nous encore? «Que le statut de Canadien français à Compton ne devint solidement établi que lorsqu'ils commencèrent à payer assidûment leur dîme et leur loyer de banc, et à assister plus régulièrement à l'office de Pâques.» Little voulait probablement nous dire que les Canadiens français une fois majoritaires, adoptèrent une certaine forme de conformisme religieux. Je crois qu'il n'a pas très bien compris tout le chapitre $V$ sur la vie culturelle dans lequel $\mathrm{j}$ 'ai abordé le thème des comportements religieux. La preuve en est la confusion de son énoncé et des notions de pascalisation qu'il assimile à l'assistance à l'office pascal. Pour l'aider à comprendre ce sujet, je lui conseille fortement la lecture des ouvrages de Gabriel Le Bras, en particulier celui qu'il a publié sur l'Église et le village.

À mon grand étonnement encore, nulle part je n'ai écrit que «l'entente règnait entre les ethnies» à Compton à l'époque de Louis-S. Saint-Laurent, enfant. Au contraire écrivais-je plutôt: «Louis Saint-Laurent grandit dans un village anglais» (p. 73) où «les deux cultures n'ont jamais été assez longtemps en présence pour s'influencer fondamentalement l'une l'autre. Elles n'ont fait que se croiser sur la route de l'histoire comme deux voitures voyageant en sens inverse» (p. 73). Est-ce assez clair?

Venons-en pour finir «aux erreurs flagrantes» que Little m'attribue. Mais avant, disposons d'une dernière erreur dont son texte est farci. J.-B.-M. Saint-Laurent, le père de Louis serait, selon notre critique, rien de moins que «le premier commerçant canadien-français estrien». Rien que cela!

Les miennes maintenant. «C'est en 1841 et non pas en 1858 que la British American Land Company perdit sa subvention», poursuit-il. Où ai-je parlé de subvention? Ce qui est écrit page 10, c'est que l'Assemblée législative du Canada-Uni avait «obtenu la rétrocession à la Couronne en 1858 de plus de la moitié des concessions originaires de la British American Land Company». Pour le profit de M. Little, je le renvoie aux Documents de la Session de l'Assemblée législative, vol. 6, p. 4.

Quant au St-Maurice and Atlantic Railway, je reconnais qu'il puisse y avoir eu confusion. Effectivement le St-Lawrence and Atlantic Railway, communément appelé Grand Tronc, passait par les Cantons de l'Est comme cela est décrit dans le détail à la page 12 de mon texte. Mais il n'était pas le seul comme semble l'affirmer Little. Le réseau ferré des Cantons comprenait certes le Grand Tronc mais aussi de multiples voies secondaires et indépendantes comme le St-Maurice, le Massawipi, le Stanstead, Shefford \& Chambly etc.

Je vous saurais gré, en terminant, messieurs, mesdames les rédacteurs, de faire parvenir copie de cette lettre à $\mathbf{M}$. Little et de publier ma mise au point 
dans un prochain numéro de votre revue. Cela me paraît justice étant donné le caractère résolument fautif du compte rendu de $\mathrm{M}$. Little. 